\title{
ON THE DIFFERENTIABILITY OF ARBITRARY REAL-VALUED SET FUNCTIONS. II( $\left.{ }^{1}\right)$
}

\author{
BY \\ HARVEL WRIGHT AND W. S. SNYDER
}

\begin{abstract}
Let $f$ be a real-valued function defined and finite on sets from a family $\mathscr{F}$ of bounded measurable subsets of Euclidean $n$-space such that if $T \in \mathscr{F}$, the measure of $T$ is equal to the measure of the closure of $T$. An earlier paper [Trans. Amer. Math. Soc. 145 (1969), 439-454] considered the questions of finiteness and boundedness of the upper and lower regular derivates of $f$ and of the existence of a unique finite derivative. The present paper is an extension of the earlier paper and considers the summability of the derivates. Necessary and sufficient conditions are given for each of the upper and lower derivates to be summable on a measurable set of finite measure. A characterization of the integral of the upper derivate is given in terms of the sums of the values of the function over finite collections of mutually disjoint sets from the family.
\end{abstract}

Introduction. This work represents a continuation of a recent paper (hereinafter referred to as Paper I) published in the Transactions [6]. Consider a family $\mathscr{F}$ of measurable subsets of Euclidean $n$-space and a real-valued function $f$ that is defined and finite on sets from $\mathscr{F}$. Paper I considered the question of finiteness and boundedness of the upper and lower derivates (see Definition 2 below) of $f$ and of the existence of a unique, finite derivative. The present paper extends the results of Paper I and considers the summability of the derivates. $\$ 1$ contains definitions and some comments on notation and $\$ 2$ contains some preliminary results to be used later. $\$ 3$ gives a necessary and sufficient condition that each of the upper derivate and lower derivate of a nonnegative function be summable on a measurable set of finite measure. $\$ 4$ removes the restriction that the function be nonnegative and $\S 5$ characterizes the integral of the upper derivate (or lower derivate) in terms of the sums of the values of the function over finite collections of mutually disjoint sets from the family $\mathscr{F}$.

1. Definitions and basic concepts. As in Paper I, this work will be restricted to Euclidean $n$-space $R_{n}$ and Lebesgue measure. The functions considered will be arbitrary real- and finite-valued set functions. The family $\mathscr{F}$ of sets on which the function is defined will be quite general and will not be required to be additive. The

Received by the editors October 2, 1970.

AMS 1970 subject classifications. Primary 28A15; Secondary 26A42.

Key words and phrases. Differentiation of set functions, regular derivate, summability, Euclidean $n$-space, Lebesgue integral, Lebesgue measure, Vitali covering.

( $\left.{ }^{1}\right)$ Research sponsored by the U.S. Atomic Energy Commission under contract with Union Carbide Corporation. 
only restriction to be placed on the family $\mathscr{F}$ is that if $T$ is a set in $\mathscr{F}$, then $T$ is measurable and the measure, $|T|$, of $T$ is equal to the measure of the closure, $\bar{T}$, of T, i.e. $|T|=|\bar{T}|$.

Definition 1. The parameter of regularity, $r(T)$, of a bounded, measurable set $T$ is defined as l.u.b. $|T| /|J|$ where $J$ denotes a cube containing $T$.

Definition 2. Let $\alpha$ represent a real number such that $0<\alpha<1$, let $\chi$ be a point in $R_{n}$, and suppose that $\mathscr{F}$ contains at least one regular $\alpha$ sequence of sets that closes down on $\chi$. The upper $(\mathscr{F}, \alpha)$ derivate of $f$ at the point $\chi$ is defined to be

$$
D^{\circ}(f, \alpha, x)=\lim \sup f(T) /|T|=\lim _{\Delta \rightarrow 0}[\text { l.u.b. } f(T) /|T|]
$$

and the lower $(\mathscr{F}, \alpha)$ derivate of $f$ at $x$ is defined to be

$$
\left.D_{0}(f, \alpha, x)=\lim \inf f(T) /|T|=\lim _{\Delta \rightarrow 0} \text { [g.l.b. } f(T) /|T|\right]
$$

where the lim sup and the $\lim$ inf are taken as $\operatorname{diam}(T) \rightarrow 0$ for sets $T$ such that $x \in T, T \in \mathscr{F}$, and $r(T)>\alpha$, and the l.u.b. and g.l.b. are taken over sets $T$ such that $x \in T, T \in \mathscr{F}, r(T)>\alpha$, and $\operatorname{diam}(T)<\Delta$.

Definition 3. The upper $(\mathscr{F}, 0)$ derivate of $f$ at the point $x$ is defined to be $D^{0}(f, x)=\lim _{\alpha \rightarrow 0+} D^{0}(f, \alpha, x)=$ l.u.b..$_{0<\alpha<1} D^{0}(f, \alpha, x)$ and the lower $(\mathscr{F}, 0)$ derivate of $f$ at $x$ is defined to be $D_{0}(f, x)=\lim _{\alpha \rightarrow 0+} D_{0}(f, \alpha, x)=$ g.l.b..$_{0<\alpha<1} D_{0}(f, \alpha, x)$.

The reader is referred to Paper I for further amplification of these definitions as well as some of their properties and implications. Paper I also contains a discussion of the Vitali covering theorem, which will be used extensively in the proofs that follow.

Script letters $\mathscr{E}$ and $\mathscr{H}$ will be used to denote finite collections of mutually disjoint sets from the family $\mathscr{F}$. A $\operatorname{dot}(\cdot)$ placed over a script letter will denote the point set obtained by the union of the sets in the collection, e.g. $\mathscr{E}=U_{T \in \mathscr{E}} T$. $\operatorname{Diam}(\mathscr{E}) \equiv \max _{T \in \mathscr{E}}[\operatorname{diam}(T)]$ and $r(\mathscr{E}) \equiv \min _{T \in \mathscr{E}} r(T)$. For any other function $f$ defined on sets $T$ in $\mathscr{E}, f(\mathscr{E}) \equiv \sum_{T \in \mathscr{E}} f(T) . S_{\alpha}$ will denote the domain of definition of the derivates above. The collection $\mathscr{F}_{\alpha}$ is defined to be the collection of all sets $T \in \mathscr{F}$ with $r(T)>\alpha$.

The notation will be the same as that in Paper I and in the interest of brevity no further discussion will be given here.

2. Preliminary results. It is well known (see, for instance, Goffman [1, pp. 219222]) that

REMARK 1. If $g(x)$ is a measurable function of $x$ that is summable on a measurable set $E$ of finite measure, then $\int_{S} g(x) d x$ is a set function that is completely additive and absolutely continuous on the class of measurable subsets $S$ of $E$.

REMARK 2. Theorem 10 of Paper I immediately guarantees the differentiability of the indefinite integral $f(T)=\int_{T \cap E} g(x) d x$ of a function $g(x)$ that is summable on a measurable set $E$. 
Furthermore, it can be shown (see, for instance, Lebesgue [3, p. 399] and Saks $[5$, p. 118]) that

REMARK 3. If $f(S)=\int_{S} g(x) d x$ where $S$ is a measurable set and where $g(x)$ is summable on $s$, then $0<\alpha<1$ implies that $D\left(f, \alpha, x_{0}\right)=g\left(x_{0}\right)$ at almost all points of the space.

LEMMA 1. Let $G$ be an open set containing a measurable set $E$ of finite measure, let $n$ and $k$ be arbitrary positive integers, and suppose $\left|E-S_{\alpha}\right|=0$. If $D^{0}(f, \alpha, x)$ is summable on $E$, then the collection $\mathscr{F}^{\prime}=\left\{T \mid T \in \mathscr{F}_{\alpha}, T \subset G\right.$, $\operatorname{diam}(T)<1 / n$ and $\left.\left|f(T)-\int_{T \cap E} D^{0}(f, \alpha, x) d x\right|<|T| / k\right\}$ covers $E$ in the sense of Vitali. A similar statement holds if $D^{0}(f, \alpha, x)$ is replaced by $D_{0}(f, \alpha, x)$.

Proof. Let $n$ and $k$ be arbitrary positive integers. Let $g(x)=D^{\circ}(f, \alpha, x)$ at each point $x \in E$ for which $D^{0}(f, \alpha, x)$ exists and is finite and let $g(x)=0$ elsewhere. Since $D^{\circ}(f, \alpha, x)$ is summable over $E, g(x)$ is summable by Remark 3, $D\left(\int_{T} g(x) d x, \alpha, x_{0}\right)=g\left(x_{0}\right)$ almost everywhere. Let $E^{\prime}$ be the subset of $E$ on which $D^{\circ}(f, \alpha, x)$ exists, is finite, and the above equality holds. Then $\left|E-E^{\prime}\right|=0$ and it suffices to show that $\mathscr{F}^{\prime}$ covers $E^{\prime}$ in the sense of Vitali. Let $x_{0}$ be an arbitrary point in $E^{\prime}$. By Remark 1 of Paper I there exists a regular $\alpha$ sequence $\left\{T_{i}\right\}$ closing down on $x_{0}$ such that $\lim _{i \rightarrow \infty} f\left(T_{i}\right) /\left|T_{i}\right|=D^{0}\left(f, \alpha, x_{0}\right)$. Consequently, there exists an integer $j_{1}>1 / n$ such that $i>j_{1}$ implies $T_{i} \subset G$, diam $\left(T_{i}\right)<1 / n$, and $\left|f\left(T_{i}\right) /\right| T_{i}\left|-D^{0}\left(f, \alpha, x_{0}\right)\right|$ $<1 / 2 k$. But

$$
D\left(\int_{T} g(x) d x, \alpha, x_{0}\right)=D\left(\int_{T \cap E} D^{0}(f, \alpha, x) d x, \alpha, x_{0}\right)=D^{0}\left(f, \alpha, x_{0}\right)
$$

and, therefore, for any regular $\alpha$ sequence closing down on $x_{0}$ (in particular for the one just mentioned) there is an integer $j_{2}$ such that $i>j_{2}$ implies $T_{i} \subset G, \operatorname{diam}\left(T_{i}\right)$ $<1 / n$, and

$$
\left|\frac{\int_{T_{\imath} \cap E} D^{\circ}(f, \alpha, x) d x}{\left|T_{i}\right|}-D^{\circ}\left(f, \alpha, x_{0}\right)\right|<\frac{1}{2 k} .
$$

Hence, for $i>\max \left(j_{1}, j_{2}\right)$

$$
\begin{aligned}
& \left|\frac{f\left(T_{i}\right)}{\left|T_{i}\right|}-\frac{\int_{T_{i} \cap E} D^{0}(f, \alpha, x) d x}{\left|T_{i}\right|}\right| \\
& \quad \leqq\left|\frac{f\left(T_{i}\right)}{\left|T_{i}\right|}-D^{0}\left(f, \alpha, x_{0}\right)\right|+\left|\frac{\int_{T_{i} \cap E} D^{0}(f, \alpha, x) d x}{\left|T_{i}\right|}-D^{0}\left(f, \alpha, x_{0}\right)\right|<\frac{1}{k}
\end{aligned}
$$

Thus at almost all points $x \in E$, there is a regular $\alpha$ sequence with the required properties closing down on $x$ and the lemma is proved for $D^{0}(f, \alpha, x)$. A similar argument holds for $D_{0}(f, \alpha, x)$.

The next lemma is similar to a result obtained by R. C. Young [7, p. 187]. 
LEMMA 2. Let $E$ be a measurable set of finite measure, let $\left|E-S_{\alpha}\right|=0$, and suppose $D^{0}(f, \alpha, x)$ is summable on $E$. Then for arbitrary positive numbers $\varepsilon, \eta$, and $\zeta$, there is a collection $\mathscr{E}$ with $r(\mathscr{E})>\alpha$, diam $(\mathscr{E})<\zeta,|E \nabla \mathscr{E}|<\eta$ and $\left|f(\mathscr{E})-\int_{E} D^{0}(f, \alpha, x) d x\right|$ $<\varepsilon$. A similar statement holds if $D^{0}(f, \alpha, x)$ is replaced by $D_{0}(f, \alpha, x)$.

Proof. Since, by Remark $1, \int_{S} D^{0}(f, \alpha, x) d x$ is a set function that is absolutely continuous on the class of measurable subsets of $E$, let $\delta$ be a number such that if $A$ is any measurable subset of $E$ with $|A|<\delta$, then $\left|\int_{A} D^{0}(f, \alpha, x) d x\right|<\varepsilon / 2$. Let $G$ be an open set such that $E \subset G$ and $|G-E|<\min (\delta / 2, \eta / 2)$. Let $k$ be an integer such that $|G| / k<\varepsilon / 2$ and let $n$ be an integer such that $1 / n<\zeta$. By Lemma 1 , the collection $\mathscr{F}^{\prime}=\left\{T \mid T \in \mathscr{F}_{\alpha}, T \subset G\right.$, diam $(T)<1 / n$, and $\left|f(T)-\int_{T \cap E} D^{0}(f, \alpha, x) d x\right|$ $<|T| \mid k\}$ covers $E$ in the sense of Vitali. By the Vitali covering theorem let $\mathscr{E}$ be a finite collection from $\mathscr{F}^{\prime}$ such that $|E-\mathscr{E}|<\min (\delta / 2, \eta / 2)$. Since $\mathscr{E} \subset G,(E \nabla \mathscr{E})$ $\subset(E-\mathscr{E}) \cup(G-E)$ and, therefore, $|E \nabla \mathscr{E}| \leqq|E-\mathscr{E}|+|G-E|<\min (\delta, \eta)$. Now since

$$
\left|f(T)-\int_{T \cap E} D^{0}(f, \alpha, x) d x\right|<\frac{|T|}{k}
$$

for each $T \in \mathscr{E}$, it follows that

$$
\begin{aligned}
\mid f(\mathscr{E}) & -\int_{E} D^{\circ}(f, \alpha, x) d x \mid \\
& \leqq\left|f(\mathscr{E})-\int_{\dot{\mathscr{\delta}} \cap E} D^{\circ}(f, \alpha, x) d x\right|+\left|\int_{\dot{\delta} \cap E} D^{\circ}(f, \alpha, x) d x-\int_{E} D^{0}(f, \alpha, x) d x\right| \\
& \leqq \frac{|\mathscr{E}|}{k}+\left|\int_{\dot{\mathscr{\delta}} \nabla E} D^{0}(f, \alpha, x) d x\right|<\frac{\varepsilon}{2}+\frac{\varepsilon}{2}=\varepsilon .
\end{aligned}
$$

A similar argument holds when $D^{\circ}(f, \alpha, x)$ is replaced by $D_{0}(f, \alpha, x)$ and the theorem is proved.

LEMMA 3. Let $E$ be a measurable set of finite measure, let $\left|E-S_{\alpha}\right|=0$, and suppose $D^{0}(f, \alpha, x)$ is summable over $E$. Then $\int_{E} D^{0}(f, \alpha, x) d x \leqq a$ for any number a which satisfies the following condition: for an arbitrary $\varepsilon>0$, there exist numbers $\zeta>0$ and $\eta>0$ with the property that for any $\mathscr{E}$ with $|\mathscr{E}-E|<\eta, r(\mathscr{E})>\alpha$, and $\operatorname{diam}(\mathscr{E})<\zeta$, there exists a subcollection $\mathscr{E}^{\prime}$ of $\mathscr{E}$ such that $\left|\mathscr{E}-\mathscr{E}^{\prime}\right|<\varepsilon$ and $f\left(\mathscr{E}^{\prime}\right)<a$.

Proof. Suppose the contrary that there exists a number $a$ and a number $\theta>0$ such that $\int_{E} D^{\circ}(f, \alpha, x) d x>a+\theta$ and such that $a$ satisfies the condition stated in the lemma. For each positive integer $n$, let $E_{n}=\left\{x \mid x \in E\right.$ and $\left.\left|D^{\circ}(f, \alpha, x)\right|<n\right\}$. Then $\lim _{n \rightarrow \infty}\left|E_{n}\right|=|E|$ and $E_{n} \subset E_{n+1}$ for each $n$. Let $N$ be an integer such that $\int_{E_{N}} D^{0}(f, \alpha, x) d x>a+\theta$. Let $\varepsilon=\theta / 4 N$. Let $\eta$ and $\zeta$ satisfy the condition for these values of $a$ and $\varepsilon$. Let $D_{N}^{0}(f, \alpha, x)=D^{0}(f, \alpha, x)$ if $D^{0}(f, \alpha, x)<N$ and $D_{N}^{0}(f, \alpha, x)=0$ if $D^{0}(f, \alpha, x) \geqq N$. By Lemma 1 of Paper I, the family $\mathscr{F}_{N}=\left\{T \mid T \in \mathscr{F}_{\alpha}\right.$ and 
$|f(T)|<N|T|\}$ covers $E_{N}$ in the sense of Vitali. Now by Lemma 2 , let $\mathscr{E}$ be a finite collection of sets from $\mathscr{F}_{N}$ with the property that $\left|E_{N} \nabla \mathscr{E}\right|<\min (\eta, \varepsilon), r(\mathscr{E})>\alpha$, $\operatorname{diam}(\mathscr{E})<\zeta$, and $\left|f(\mathscr{E})-\int_{E_{N}} D^{0}(f, \alpha, x) d x\right|<\theta / 2$. Thus

$$
f(\mathscr{E})>\int_{E_{N}} D^{0}(f, \alpha, x) d x-\frac{\theta}{2}>a+\theta-\frac{\theta}{2}=a+\frac{\theta}{2} .
$$

Now $|\mathscr{E}-E|<\left|\mathscr{E}-E_{N}\right|<\eta, r(\mathscr{E})>\alpha$, diam $(\mathscr{E})<\zeta$ and, therefore $\mathscr{E}$ satisfies the condition stated in the lemma. But if $\mathscr{E}^{\prime}$ is a subcollection of $\mathscr{E}$ such that $\left|\mathscr{E}^{\prime}-\mathscr{E}^{\prime}\right|<\varepsilon$ then $f\left(\mathscr{E}-\mathscr{E}^{\prime}\right) \leqq N\left|\mathscr{E}^{\prime}-\mathscr{E}^{\prime}\right|<N \varepsilon<N \theta / 4 N=\theta / 4$ and since $f\left(\mathscr{E}-\mathscr{E}^{\prime}\right)=f(\mathscr{E})-f\left(\mathscr{E}^{\prime}\right)$ it follows that

$$
f\left(\mathscr{E}^{\prime}\right)=f(\mathscr{E})-f\left(\mathscr{E}-\mathscr{E}^{\prime}\right)>f(\mathscr{E})-\theta / 4>a+\theta / 2-\theta / 4>a .
$$

But this contradicts the hypothesis that $a$ satisfies the condition stated in the lemma and, consequently, the lemma is proved.

3. Summability of the derivates of nonnegative functions. This section contains theorems giving conditions which are necessary and sufficient for the upper derivate and also for the lower derivate of a nonnegative function to be summable on a measurable set of finite measure.

THEOREM 1. Let $f$ be nonnegative, let $E$ be a measurable set with finite measure, and assume $\left|E-S_{\alpha}\right|=0$. A necessary and sufficient condition that $D^{0}(f, \alpha, x)$ be summable over $E$ is that there is a number $a>0$ such that for every $\varepsilon>0$, there exist numbers $\zeta>0$ and $\eta>0$ with the property that for any $\mathscr{E}$ with $|\mathscr{E}-E|<\eta, r(\mathscr{E})>\alpha$, and diam $(\mathscr{E})<\zeta$, there exists a subcollection $\mathscr{E}^{\prime}$ of $\mathscr{E}$ such that $\left|\mathscr{E}^{\prime}-\mathscr{E}^{\prime}\right|<\varepsilon$ and $f\left(\mathscr{E}^{\prime}\right) \leqq a$.

Proof. To prove necessity, assume $D^{\circ}(f, \alpha, x)$ is summable over $E$. Thus assume $\int_{E} D^{0}(f, \alpha, x) d x=b$. The proof will be completed by showing that

REMARK 4. If $D^{0}(f, \alpha, x)$ is summable on a measurable set $E$ of finite measure, then any value for $a$ which is greater than $\int_{E} D^{\circ}(f, \alpha, x) d x$ will satisfy the condition of the theorem.

Proof. Let $\theta$ be an arbitrary positive number and let $a=b+\theta$. Suppose that the condition does not hold for this value of $a$. Let $\varepsilon$ be a positive number such that no values of $\zeta$ and $\eta$ exist which satisfy the condition. For each positive integer $n$, let $D_{n}^{0}(f, \alpha, x)=D^{0}(f, \alpha, x)$ if $D^{0}(f, \alpha, x)<n$ and equal 0 otherwise and let $E_{n}=\left\{x \mid x \in E\right.$ and $\left.D_{n}^{0}(f, \alpha, x)=D^{0}(f, \alpha, x)\right\}$. Since $D^{\circ}(f, \alpha, x)$ is summable on $E$, it is finite almost everywhere on $E$ and, therefore, there is an integer $N$ such that $\left|E-E_{N}\right|<\varepsilon / 4$. For each integer $m$, let $E_{N}^{m}=\left\{x \mid x \in E_{N}\right.$ and for any set $T$ such that $x \in T$, diam $(T)<1 / m$, and $r(T)>\alpha$, it is true that $f(T)<N|T|\}$. It follows that $E_{N}^{m} \subset E_{N}^{m+1}$ for each integer $m$, and $\lim _{m \rightarrow \infty} E_{N}^{m}=E_{N}$. Let $m_{0}$ be an integer such that [5, p. 47] $\left|E_{N}^{m_{0}}\right|_{e}>\left|E_{N}\right|-\varepsilon / 4$. Let $M$ be an integer such that $M>\max \left(m_{0}, 4 / \varepsilon\right)$. For each integer $m>M$, let $\zeta=\eta=1 / m$. Since the condition is assumed not to hold for 
these values of $a$ and $\varepsilon$, there is a collection $\mathscr{E}_{m}$ such that $\left|\mathscr{E}_{m}-E\right|<1 / m, r\left(\mathscr{E}_{m}\right)>\alpha$, and $\operatorname{diam}\left(\mathscr{E}_{m}\right)<1 / m$, but such that if $\mathscr{E}_{m}^{\prime}$ is a subcollection of $\mathscr{E}_{m}$ for which $\left|\mathscr{E}_{m}-\mathscr{E}_{m}^{\prime}\right|$ $<\varepsilon$, then $f\left(\mathscr{E}_{m}^{\prime}\right)>a=b+\theta$. Now delete all sets $T \in \mathscr{E}_{m}$ that do not intersect $E_{N}^{m}$ to obtain the collection $\mathscr{E}_{m}^{\prime}$. For each integer $m,\left|\mathscr{E}_{m}-\mathscr{E}_{m}^{\prime}\right|<\varepsilon$. To see this, observe that $\mathscr{E}_{m}-\mathscr{E}_{m}^{\prime} \subset\left(\mathscr{E}_{m}-E\right) \cup\left(E-\mathscr{E}_{m}^{\prime}\right)$. If a point of $\mathscr{E}_{m}$ was deleted, then it was not in $E_{N}^{m}$ so that $\left(\mathscr{E}_{m}-\mathscr{E}_{m}^{\prime}\right) \subset\left(\mathscr{E}_{m}-E\right) \cup\left(E-E_{N}^{m}\right)$. Let $A=\mathscr{E}_{m}-E$ and let $B=\left(\mathscr{E}_{m}-\mathscr{E}_{m}^{\prime}\right)-A$. Then $B \subset\left(E-E_{N}^{m}\right)$ and $B \cap E_{N}^{m}=\varnothing$. But $\left(B \cup E_{N}^{m}\right) \subset E$ and it follows that $|B| \leqq|E|-\left|E_{N}^{m}\right|_{e}$. Thus

$$
|B| \leqq\left(|E|-\left|E_{N}\right|\right)+\left(\left|E_{N}\right|-\left|E_{N}^{m_{0}}\right|_{e}\right)<\varepsilon / 4+\varepsilon / 4=\varepsilon / 2
$$

and hence

$$
\left|\mathscr{E}_{m}-\mathscr{E}_{m}^{\prime}\right| \leqq\left|\mathscr{E}_{m}-E\right|+|B|<1 / m+\varepsilon / 2<\varepsilon / 4+\varepsilon / 2<\varepsilon
$$

Now each set $T$ in $\mathscr{E}_{m}^{\prime}$ intersects $E_{N}^{m}$ and, therefore, if $m>m_{0}$ it follows from the definition of $E_{N}^{m}$ that $f(T)<N|T|$. For each integer $m>M$, define the point function $g_{m}(x)$ by $g_{m}(x)=f(T) /|T|$ if $x \in \mathscr{E}_{m}^{\prime}$, and $g_{m}(x)=0$ otherwise. Then for each $m>M, 0 \leqq g_{m}(x)<N$. Using the function $g_{m}(x)$, the number $f\left(\mathscr{E}_{m}^{\prime}\right)$ can be expressed as

$$
\begin{aligned}
f\left(\mathscr{E}_{m}^{\prime}\right) & =\int_{\ddot{\mathscr{E}}_{m}^{\prime}} g_{m}(x) d x=\int_{\dot{\mathscr{E}}_{m}^{\prime} \cap E} g_{m}(x) d x+\int_{\ddot{\mathscr{E}}_{m}^{\prime}-E} g_{m}(x) d x \\
& \leqq \int_{\dot{\mathscr{E}}_{m}^{\prime} \cap E} g_{m}(x) d x+N\left|\mathscr{E}_{m}^{\prime}-E\right| .
\end{aligned}
$$

But since $\left(\mathscr{E}_{m}^{\prime}-E\right) \subset\left(\mathscr{E}_{m}-E\right)$ then $\left|\mathscr{E}_{m}^{\prime}-E\right| \leqq\left|\mathscr{E}_{m}-E\right|<1 / m$. Thus

$$
f\left(\mathscr{E}_{m}^{\prime}\right) \leqq \int_{\dot{\delta}_{m}^{\prime} \cap E} g_{m}(x) d x+N / m \text { for any } m>M .
$$

Hence

$$
\int_{\dot{\mathscr{E}}_{m}^{\prime} \cap E} g_{m}(x) d x \geqq f\left(\mathscr{E}_{m}^{\prime}\right)-N / m>a-N / m=b+\theta-N / m .
$$

Since $g_{m}(x)=0$ for $x$ not in $\mathscr{E}_{m}^{\prime}$, it follows that

$$
\int_{\dot{\mathscr{E}}_{m}^{\prime} \cap E} g_{m}(x) d x=\int_{E} g_{m}(x) d x
$$

Now $g_{m}(x)$ is bounded on the set $E$ with $|E|<+\infty$ by the integrable function having the constant value $N$ and, therefore [5, p. 29]

$$
\int_{E} \lim \sup _{m} g_{m}(x) d x \geqq \lim \sup _{m} \int_{E} g_{m}(x) d x=\lim \sup _{m} \int_{\dot{\delta}_{m}^{\prime} \cap E} g_{m}(x) d x \geqq b+\theta \text {. }
$$

But by the definition of $D^{0}(f, \alpha, x), \lim \sup _{m} g_{m}(x) \leqq D^{0}(f, \alpha, x)$ so that

$$
\int_{E} D^{0}(f, \alpha, x) d x \geqq \int_{E} \limsup _{m} g_{m}(x) d x \geqq b+\theta
$$


which is a contradiction that $\int_{E} D^{0}(f, \alpha, x) d x=b$ and completes the proof of Remark 4 as well as the necessity of the theorem.

To prove sufficiency, assume the condition holds. Suppose $\int_{E} D^{0}(f, \alpha, x) d x$ $=+\infty$. The proof will be completed by showing that this supposition leads to a contradiction. Since $f$ is nonnegative, then either (1) there exists a subset $E_{\infty}$ of $E$ with $\left|E_{\infty}\right|>0$ and $D^{0}(f, \alpha, x)=+\infty$ for $x \in E_{\infty}$ or else (2) $D^{0}(f, \alpha, x)$ is finite almost everywhere on $E$. These two cases will be considered separately.

Case 1. Suppose $D^{0}(f, \alpha, x)=+\infty$ at each point $x \in E_{\infty}$ where $\left|E_{\infty}\right|>0$ and suppose that $a$ is a number which satisfies the condition. Let $\varepsilon=\left|E_{\infty}\right| / 4$ and let $\eta$ and $\zeta$ satisfy the condition for this value of $\varepsilon$. Let $G$ be an open set containing $E_{\infty}$ and such that $\left|G-E_{\infty}\right|<\eta$. By Lemma 1 the family $\mathscr{F}^{\prime}=\left\{T \mid T \in \mathscr{F}_{\alpha}\right.$, diam $(T)<\zeta$, $T \subset G$ and $\left.f(T)>\left(2 a|| E_{\infty} \mid\right)|T|\right\}$ covers $E_{\infty}$ in the sense of Vitali. By the Vitali covering theorem, there exists a collection $\mathscr{E}$ of sets from $\mathscr{F}^{\prime}$ such that $\left|E_{\infty}-\mathscr{E}\right|$ $<\left|E_{\infty}\right| / 4=\varepsilon$. Now $|\mathscr{E}-E| \leqq\left|G-E_{\infty}\right|<\eta$, and $|\mathscr{E}| \geqq\left|E_{\infty} \cap \mathscr{E}\right| \geqq\left|E_{\infty}\right|-\left|E_{\infty}-\mathscr{E}\right|$ $\geqq 3\left|E_{\infty}\right| / 4=3 \varepsilon$. Hence if $\mathscr{E}^{\prime}$ is a subcollection of $\mathscr{E}$ such that $\left|\mathscr{E}-\mathscr{E}^{\prime}\right|<\varepsilon$, then $\left|\mathscr{E}^{\prime}\right| \geqq\left|\mathscr{E}^{\mathscr{E}}\right|-\left|\mathscr{E}^{\prime}-\mathscr{E}^{\prime}\right|>2 \varepsilon=\left|E_{\infty}\right| / 2$ and, consequently, $f\left(\mathscr{E}^{\prime}\right)>\left(2 a|| E_{\infty} \mid\right) \cdot\left|\mathscr{E}^{\prime}\right|>a$ which contradicts the hypothesis that the condition holds.

Case 2. Suppose $D^{0}(f, \alpha, x)$ is finite almost everywhere on $E$. For each integer $n$, let $E_{n}=\left\{x \mid x \in E\right.$ and $\left.D^{0}(f, \alpha, x)<n\right\}$. Then $E_{n} \subset E_{n+1}$ for each $n$ and $\lim _{n \rightarrow \infty}\left|E_{n}\right|$ $=|E|$. Let $a$ satisfy the condition of the theorem and let $\theta$ be an arbitrary positive number. Since $D^{0}(f, \alpha, x)$ is assumed not to be summable on $E$, there exists an integer $N$ such that $\int_{E_{N}} D^{0}(f, \alpha, x) d x>a+\theta$. It follows from Lemma 3 that this is a contradiction and therefore the theorem is proved.

COROLlary 1. If $f$ is nonnegative and absolutely continuous, then the condition of Theorem 1 is satisfied and $D^{\circ}(f, \alpha, x)$ is summable on $E$.

Proof. Since $f$ is absolutely continuous, there is a $\delta>0$ such that if $\mathscr{E}$ has $|\mathscr{E}|<\delta$, then $f(\mathscr{E})<1$. If $a=2(|E|+\delta) / \delta$, then this value of $a$ satisfies the condition of Theorem 1. To see this, let $\varepsilon>0$ be arbitrary. Let $\eta=\delta$ and let $\zeta$ be any number such that $\operatorname{diam}(T)<\zeta$ implies $|T|<\delta / 2$. Now let $\mathscr{E}$ be any collection such that $|\mathscr{E}-E|<\eta$ and diam $(\mathscr{E})<\zeta$. Group the sets in the finite collection $\mathscr{E}$ into subcollections $\mathscr{E}_{i}$ with the property that $\delta / 2<\left|\mathscr{E}_{i}\right|<\delta$ for each value of $i$ except possibly the last value which may have $0<\left|\mathscr{E}_{i}\right|<\delta$. Since $|\mathscr{E}|-|E| \leqq|\mathscr{E}-E|<\delta$, then $|\mathscr{E}|<|E|+\delta$ and it follows that there are at most $(|E|+\delta) / 0.5 \delta=2(|E|+\delta) / \delta$ values of $i$. But by the absolute continuity of $f, f\left(\mathscr{E}_{i}\right)<1$ for each $i$ and thus $f(\mathscr{E})$ $<(2(|E|+\delta) / \delta) \cdot 1=a$. Thus by Theorem $1, D^{\circ}(f, \alpha, x)$ is summable on $E$.

THEOREM 2. Let $f$ be nonnegative and suppose there is a number $\beta$ with $0<\beta<1$ such that $0<\alpha<\beta$ implies $\left|E-S_{\alpha}\right|=0$. A necessary and sufficient condition that $D^{0}(f, x)$ be summable on $E$ is that the a in Theorem 1 can be taken independent of $\alpha$.

Proof. To prove necessity, assume $D^{0}(f, x)$ is summable on $E$. Since $D^{0}(f, \alpha, x)$ is a nonincreasing function of $\alpha, D^{0}(f, \alpha, x) \leqq D^{0}(f, x)$ for any $\alpha$ where $0<\alpha<\beta$ 
and, therefore, $D^{0}(f, \alpha, x)$ is summable on $E$. Let $\theta>0$ be arbitrary and let $a=\int_{E} D^{\circ}(f, x) d x+\theta$. Then by Remark 4, this value of $a$ will satisfy the condition for any $\alpha$ and hence $a$ can be chosen independent of $\alpha$.

To prove sufficiency, suppose $a$ can be chosen independent of $\alpha$. By Lemma 3, $\int_{E} D^{\circ}(f, \alpha, x) d x \leqq a$ for every $\alpha$ such that $0<\alpha<\beta$ and for any $a$ which satisfies the condition. Then it follows $[5$, p. 28$]$ that

$$
\int_{E} D^{0}(f, x) d x=\int_{E} \lim _{\alpha \rightarrow 0} D^{\circ}(f, \alpha, x) d x=\lim _{\alpha \rightarrow 0} \int_{E} D^{0}(f, \alpha, x) d x \leqq a .
$$

Thus $D^{0}(f, x)$ is summable over $E$.

THEOREM 3. Let $f$ be nonnegative, let $E$ be measurable with $|E|<+\infty$, and assume $\left|E-S_{\alpha}\right|=0$. Then a necessary and sufficient condition that $D_{0}(f, \alpha, x)$ be summable on $E$ is that there exists a number a with the property that for each integer $n$, there is an $\mathscr{E}_{n}$ such that $\left|\mathscr{E}_{n} \nabla E\right|<1 / n, r\left(\mathscr{E}_{n}\right)>\alpha$, diam $\left(\mathscr{E}_{n}\right)<1 / n$ and $f\left(\mathscr{E}_{n}\right) \leqq a$.

Proof. To prove sufficiency, assume that the condition holds. It may be clearly assumed that $E \subset S_{\alpha}$. For each positive integer $n$, let $\zeta=\eta=1 / n$. Now the condition assures the existence of a collection $\mathscr{E}_{n}$ such that $r\left(\mathscr{E}_{n}\right)>\alpha$, diam $\left(\mathscr{E}_{n}\right)<1 / n,\left|\mathscr{E}_{n} \nabla E\right|$ $<1 / n$, and $f\left(\mathscr{E}_{n}\right) \leqq a$. Let $B=\lim \sup _{n} \mathscr{E}_{n}$. It follows that $|B|=|E|$. To see this suppose the contrary, i.e., suppose there is a set $A \subset(E-B)$ such that $|A|>0$. Let $N$ be an integer such that $N>2 /|A|$. Then for each $n>N,\left|A-\mathscr{E}_{n}\right| \leqq\left|E-\mathscr{E}_{n}\right|<1 / n$ $<1 / N$. But $\left|A \cap \mathscr{E}_{n}\right| \geqq|A|-\left|A-\mathscr{E}_{n}\right|>2 / N-1 / N=1 / N$ and it follows that [5, p. 8] $\left|\lim \sup _{n}\left(\mathscr{E}_{n} \cap A\right)\right|>1 / N$. Thus $\lim \sup _{n}(\mathscr{E} \cap A)=B \cap A$ is not empty which contradicts $A \subset(E-B)$. Hence $|B|=|E|$. Now consider the point function $g_{n}(x)$ defined by $g_{n}(x)=f(T) /|T|$ if $x \in \mathscr{E}_{n}$ and $g_{n}(x)=0$ otherwise. Since $g_{n}(x)$ is measurable for each $n$, it follows that $\lim _{\inf _{n}} g_{n}(x)$ is also a measurable function. Now

$$
\int_{E} g_{n}(x) d x=\int_{E \cap \dot{\delta}_{n}} g_{n}(x) d x \leqq \int_{\dot{\delta}_{n}} g_{n}(x) d x=\sum_{T \in \mathscr{\delta}_{n}} \frac{f(T)}{|T|} \cdot|T|=f\left(\mathscr{E}_{n}\right) \leqq a
$$

and it follows that [5, p. 29] $\int_{E} \liminf _{n} g_{n}(x) d x \leqq \liminf \int_{E} g_{n}(x) d x \leqq a$. But $D_{0}(f, \alpha, x) \leqq \lim \inf _{n} g_{n}(x)$ for each $x \in B$ and thus $\int_{E} D_{0}(f, \alpha, x) \leqq a$ and the sufficiency is proved.

To prove necessity, assume $D_{0}(f, \alpha, x)$ is summable on $E$. Let $\int_{E} D_{0}(f, \alpha, x) d x$ $=b$. Let $\theta>0$ be arbitrary and let $a=b+\theta$. Let $n$ be an arbitrary positive integer. By Lemma 2 , let $\mathscr{E}_{n}$ be a collection such that $r\left(\mathscr{E}_{n}\right)>\alpha$, diam $\left(\mathscr{E}_{n}\right)<1 / n,\left|\mathscr{E}_{n} \nabla E\right|$ $<1 / n$, and $\left|f\left(\mathscr{E}_{n}\right)-\int_{E} D_{0}(f, \alpha, x) d x\right|<\theta / 2$. Then $f\left(\mathscr{E}_{n}\right)<\int_{E} D_{0}(f, \alpha, x) d x+\theta / 2$ $=a-\theta+\theta / 2<a$ and the necessity is proved.

4. Summability of the derivates of arbitrary functions. The above theorems regarding the summability of $D^{\circ}(f, \alpha, x)$ and $D_{0}(f, \alpha, x)$ have been restricted to nonnegative functions. For an arbitrary function, Theorems $1(\mathrm{~g})$ and $1(\mathrm{~h})$ of Paper I can be used in connection with the above theorems for nonnegative functions as stated in 
THeOrem 4. For an arbitrary function $f, D^{\circ}(f, \alpha, x)$ will be summable on $E$ if and only if each of $D^{0}\left(f^{+}, \alpha, x\right)$ and $D_{0}\left(f^{-}, \alpha, x\right)$ is summable on $E$ and $D_{0}(f, \alpha, x)$ will be summable on $E$ if and only if each of $D_{0}\left(f^{+}, \alpha, x\right)$ and $D^{\circ}\left(f^{-}, \alpha, x\right)$ is summable on $E$.

Proof. By Theorem 1(f) of Paper I, $D^{\circ}(f, \alpha, x)=D^{\circ}\left(f^{+}, \alpha, x\right)-D_{0}\left(f^{-}, \alpha, x\right)$ and at most one of the terms on the right can be nonzero. Let $E^{+}=\{x \mid x \in E$ and $\left.D^{0}\left(f^{+}, \alpha, x\right)>0\right\}$, let $E^{-}=\left\{x \mid x \in E\right.$ and $\left.D_{0}\left(f^{-}, \alpha, x\right)>0\right\}$, and let $E^{0}=\{x \mid x \in E$ and $\left.D^{0}\left(f^{+}, \alpha, x\right)=D_{0}\left(f^{-}, \alpha, x\right)=0\right\}$. Then $E=E^{+} \cup E^{-} \cup E^{0}$ and these three sets are pairwise disjoint. Now $\int_{E} D^{0}(f, \alpha, x)=\int_{E^{+}} D^{0}\left(f^{+}, \alpha, x\right) d x-\int_{E^{-}} D_{0}\left(f^{-}, \alpha, x\right) d x$ because

and

$$
\int_{E^{+}} D^{0}\left(f^{+}, \alpha, x\right) d x=\int_{E^{+}} D^{0}(f, \alpha, x) d x
$$

$$
-\int_{E^{-}} D_{0}\left(f^{-}, \alpha, x\right) d x=\int_{E^{-}} D^{0}(f, \alpha, x) d x
$$

and, therefore, it follows that $D^{0}(f, \alpha, x)$ is summable on $E$ if and only if each of $D^{0}\left(f^{+}, \alpha, x\right)$ and $D_{0}\left(f^{-}, \alpha, x\right)$ is summable on $E$. A similar argument holds for the lower derivate.

A necessary and sufficient condition that all of the derivates of an arbitrary function be summable can now be stated as an immediate consequence of Theorem 1.

THEOREM 5. Let $f$ be an arbitrary real-valued set function, let $E$ be a measurable set with finite measure and assume $\left|E-S_{\alpha}\right|=0$. A necessary and sufficient condition that both $D^{0}(f, \alpha, x)$ and $D_{0}(f, \alpha, x)$ be summable on $E$ is that there is a number $a>0$ such that for every $\varepsilon>0$, there exist numbers $\zeta>0$ and $\eta>0$ with the property that for any $\mathscr{E}$ with $|\mathscr{E}-E|<\eta, r(\mathscr{E})>\alpha$, and $\operatorname{diam}(\mathscr{E})<\zeta$, there exists a subcollection $\mathscr{E}^{\prime}$ of $\mathscr{E}$ such that $\left|\mathscr{E}-\mathscr{E}^{\prime}\right|<\varepsilon$ and $|f|\left(\mathscr{E}^{\prime}\right) \leqq a$.

This theorem simply states the condition that the upper derivate of the nonnegative function $|f|$ be summable on $E$ and is essentially a restatement of Theorem 1 for the nonnegative function $|f|$.

5. Characterization of the integral. In view of Lemma 3 and Remark 4, the value of the integral of $D^{\circ}(f, \alpha, x)$ can be seen to be the infimum of values of $a$ which satisfy the condition of Theorem 1 . It is therefore possible to specify the value of the integral explicitly in terms of the values of the function $f$ on sets in finite collections of sets from $\mathscr{F}$. This value is stated in

THEOREM 6. Let $f$ be nonnegative and assume that $\left|E-S_{\alpha}\right|=0$. If $D^{\circ}(f, \alpha, x)$ is summable on $E$, then

$$
\lim _{k \rightarrow \infty}\left(\lim _{n \rightarrow \infty}\left[\sup \left\{\inf f\left(\mathscr{E}^{\prime}\right)\right\}\right]\right)=\int_{E} D^{0}(f, \alpha, x) d x,
$$


where the supremum is taken over collections $\mathscr{E}$ such that $|\mathscr{E} \nabla E|<1 / n, r(\mathscr{E})>\alpha$, and diam $(\mathscr{E})<1 / n$, and where the infimum is taken over subcollections $\mathscr{E}^{\prime}$ of $\mathscr{E}$ for which $\left|\mathscr{E}-\mathscr{E}^{\prime}\right|<1 / k$.

Proof. Let $\int_{E} D^{0}(f, \alpha, x) d x=b$. First it will be shown that the expression on the left of the equation (1) is $\leqq b$. To see this, let $\theta>0$ be arbitrary and let $a=b+\theta$. Since $D^{0}(f, \alpha, x)$ is summable on $E$, then by Remark 4 this value of $a$ satisfies the condition of Theorem 1 . Let $k_{0}$ be an arbitrary positive integer and let $\varepsilon$ be a positive number such that $\varepsilon<1 / k_{0}$. Let $\zeta>0$ and $\eta>0$ satisfy the condition of Theorem 1 for this value of $\varepsilon$. Let $n>\max (1 / \zeta, 1 / \eta)$. Now let $\mathscr{E}$ be any collection such that $|\mathscr{E} \nabla E|<1 / n, r(\mathscr{E})>\alpha$, and diam $(\mathscr{E})<1 / n$. The condition of Theorem 1 guarantees that there exists a subcollection $\mathscr{E}^{\prime}$ of $\mathscr{E}$ such that $\left|\mathscr{E}^{\prime}-\mathscr{E}^{\prime}\right|<\varepsilon<1 / k_{0}$ and $f\left(\mathscr{E}^{\prime}\right) \leqq a$. Therefore, $\inf f\left(\mathscr{E}^{\prime}\right) \leqq a$ where the infimum is taken over all subcollections $\mathscr{E}^{\prime}$ of $\mathscr{E}$ for which $\left|\mathscr{E}-\mathscr{E}^{\prime}\right|<\varepsilon$. But this is true for an arbitrary $\mathscr{E}$ subject to $|\mathscr{E} \nabla E|<1 / n$, $r(\mathscr{E})>\alpha$, and therefore, $\sup \left\{\inf f\left(\mathscr{E}^{\prime}\right)\right\} \leqq a$ where the supremum is taken over all collections $\mathscr{E}$ for which $|\mathscr{E} \nabla E|<1 / n, r(\mathscr{E})>\alpha$, and therefore $\lim _{n \rightarrow \infty}\left[\sup \left\{\inf f\left(\mathscr{E}^{\prime}\right)\right\}\right]$ $\leqq a$. Now $k_{0}$ was an arbitrary positive integer so that $\lim _{k \rightarrow \infty}\left(\lim _{n \rightarrow \infty}\left[\sup \left\{\inf f\left(\mathscr{E}^{\prime}\right)\right\}\right]\right)$ $\leqq a=b+\theta$. Thus the expression on the left of equation (1) is $\leqq b+\theta$ for an arbitrary $\theta>0$ and, therefore, is $\leqq b$.

Next it will be shown that the expression is $\geqq b$. Suppose the contrary, i.e., suppose there exists a number $\theta>0$ such that the expression $=b-\theta$. From the definition of limit, there is an integer $k_{0}$ such that for $k>k_{0}, \lim _{n \rightarrow \infty}\left[\sup \left\{\inf f\left(\mathscr{E}^{\prime}\right)\right\}\right]$ $<b-3 \theta / 4$ and, for this value of $k_{0}$, there is an integer $n_{0}$ such that $n>n_{0}$ implies $\sup \left\{\inf f\left(\mathscr{E}^{\prime}\right)\right\}<b-\theta / 2$. Let $a=b-\theta / 2$ and $\varepsilon=1 / k_{0}$. Let $\eta<1 / n_{0}$ and $\zeta<1 / n_{0}$. Consequently, for any collection $\mathscr{E}$ such that $|\mathscr{E} \nabla E|<\eta, r(\mathscr{E})>\alpha$, and diam $(\mathscr{E})$ $<\zeta$, there is a subcollection $\mathscr{E}^{\prime}$ of $\mathscr{E}$ for which $\left|\mathscr{E}^{\prime}-\mathscr{E}^{\prime}\right|<\varepsilon$ and $f\left(\mathscr{E}^{\prime}\right) \leqq b-\theta / 2<b$. Therefore, by Lemma $3, \int_{E} D^{0}(f, \alpha, x) d x \leqq a=b-\theta / 2$. But this is a contradiction which arises from the supposition that the expression is $<b$ and the theorem is proved.

It is of interest to compare Theorem 6 with results obtained by Hartnett and Kruse [2] who studied the case of a nonnegative function $\lambda$ defined on a family of subsets of a metric space. The case of Euclidean space and Lebesgue measure satisfies their condition. They define an upper measure $m^{0}$ [2, p. 189], determined by the function $\lambda$, by using open coverings directed by refinement. There is the provision in Theorem 6 of this paper for the deletion of sets on which the function $\lambda$ is very large, provided the total area of the sets deleted is sufficiently small. However, Hartnett and Kruse have no provision for such deletion and, consequently, it is possible for a single point to have positive upper measure. This leads to difficulty, as pointed out by Pauc [4], who gave a counterexample to Theorem 12 of Hartnett and Kruse [2, p. 199].

The provision for deleting a small amount of area from $\mathscr{E}$ avoids the above 
difficulty and eliminates the necessity of placing restrictions on the function under consideration.

The last result to be included in this paper is a condition that the value of a function on a set $T$ in $\mathscr{F}$ be the integral of its derivative over $T$.

LEMma 4. Let $G$ be an open set such that $\left|G-S_{\alpha}\right|=0$. If $f$ satisfies the condition that for an arbitrary $\varepsilon>0$ there is a $\Delta>0$ such that $(\mathscr{E} \cup \dot{\mathscr{H}}) \subset G$ and $|\mathscr{E} \nabla \dot{\mathscr{H}}|<\Delta$ imply that $|f(\mathscr{E})-f(\mathscr{H})|<\varepsilon$, then $f$ is absolutely continuous on $G$.

Proof. Let $\varepsilon>0$ be given. Let $\Delta$ satisfy the condition $(\mathscr{E} \cup \dot{\mathscr{H}}) \subset G$ and $|\mathscr{E} \nabla \dot{\mathscr{H}}|$ $<\Delta$ imply that $|f(\mathscr{E})-f(\mathscr{H})|<\varepsilon / 2$. Let $T_{0}$ be any set in $\mathscr{F}$ for which $\left|T_{0}\right|<\Delta / 2$ and $\left|f\left(T_{0}\right)\right|<\varepsilon / 2$. To see that such a set $T_{0}$ exists, let $T_{0}$ and $T_{1}$ be disjoint sets such that $\left|T_{0}\right|<\Delta / 4$. Let $\mathscr{E}_{0}$ be the collection consisting of the two sets $T_{0}$ and $T_{1}$ and let $\mathscr{E}_{1}$ be the collection whose only element is the set $T_{1}$. Then since $\left|\mathscr{E}_{0} \nabla \mathscr{E}_{1}\right|=\left|\mathscr{E}_{0}-\mathscr{E}_{1}\right|$ $=\left|T_{0}\right|<\Delta$, it follows from the hypothesis that $\left|f\left(\mathscr{E}_{0}\right)-f\left(\mathscr{E}_{1}\right)\right|<\varepsilon / 2$. But $\left|f\left(\mathscr{E}_{0}\right)-f\left(\mathscr{E}_{1}\right)\right|$ $=\left|f\left(T_{0}\right)\right|<\varepsilon / 2$ and, therefore, the existence of a set $T_{0}$ satisfying the condition that $\left|T_{0}\right|<\Delta / 2$ and $\left|f\left(T_{0}\right)\right|<\varepsilon / 2$ is established. Now let $\mathscr{H}$ be the collection whose only element is the set $T_{0}$ and let $\mathscr{E}$ be any collection such that $\mathscr{E} \subset G$ and $|\mathscr{E}|<\Delta / 2$. Then $|\dot{\mathscr{E}} \nabla \dot{\mathscr{H}}| \leqq|\mathscr{E}|+|\dot{\mathscr{H}}|<\Delta$ and, by the hypothesis, $|f(\mathscr{E})-f(\mathscr{H})|<\varepsilon / 2$. Therefore, $|f(\mathscr{E})|-|f(\mathscr{H})| \leqq|f(\mathscr{E})-f(\mathscr{H})|<\varepsilon / 2$ and hence $|f(\mathscr{E})|<\varepsilon / 2+|f(\mathscr{H})|<\varepsilon / 2+\varepsilon / 2=\varepsilon$. Since $\mathscr{E}$ was arbitrary, $f$ is absolutely continuous.

THEOREM 7. Let $f$ be nonnegative, let $G$ be an open set, and assume $\left|G-S_{\alpha}\right|=0$. $A$ necessary and sufficient condition that $D(f, \alpha, x)$ exists on $G \cap S_{\alpha}$, is summable on $G$, and $T \subset G$ implies $f(T)=\int_{T} D(f, \alpha, x) d x$ is that for an arbitrary $\varepsilon>0$, there exists a $\Delta>0$ such that $|\mathscr{E} \nabla \dot{\mathscr{H}}|<\Delta$ implies $|f(\mathscr{E})-f(\mathscr{H})|<\varepsilon$.

To prove sufficiency assume the condition holds. Then the condition of Theorem 10 of Paper I is satisfied and hence $D(f, \alpha, x)$ exists and is finite almost everywhere on $G$. Also, by Lemma $4, f$ is absolutely continuous on $G$ and, therefore, by Corollary $1, D(f, \alpha, x)$ is summable on $G$. Define $g(T)=\int_{T} D(f, \alpha, x) d x$. Let $T_{0}$ be an arbitrary set in $\mathscr{F}$ such that $T_{0} \subset G$. It suffices to show that $f\left(T_{0}\right)=g\left(T_{0}\right)$. Let $\varepsilon>0$ be arbitrary. Let $\Delta$ satisfy the condition that $|\mathscr{E} \nabla \dot{\mathscr{H}}|<\Delta$ implies $|f(\mathscr{E})-f(\mathscr{H})|$ $<\varepsilon / 2$. By Lemma 2, there exists a collection $\mathscr{E}$ such that $\left|T_{0} \nabla \mathscr{E}\right|<\Delta$ and

Then

$$
\left|f(\mathscr{E})-\int_{T_{0}} D(f, \alpha, x) d x\right|=\left|f(\mathscr{E})-g\left(T_{0}\right)\right|<\varepsilon / 2
$$

$$
\left|f\left(T_{0}\right)-g\left(T_{0}\right)\right| \leqq\left|f\left(T_{0}\right)-f(\mathscr{E})\right|+\left|f(\mathscr{E})-g\left(T_{0}\right)\right|<\varepsilon / 2+\varepsilon / 2=\varepsilon .
$$

For any set $T_{0} \in \mathscr{F}$ such that $T_{0} \subset G$, it has been shown that $\left|f\left(T_{0}\right)-g\left(T_{0}\right)\right|<\varepsilon$ for an arbitrary positive number $\varepsilon$ and, consequently, $f\left(T_{0}\right)=g\left(T_{0}\right)$.

To prove necessity, assume $D(f, \alpha, x)$ exists on $G \cap S_{\alpha}$, is summable on $G$, and $T \subset G$ implies $f(T)=\int_{T} D(f, \alpha, x) d x$. By Remark $1, f$ is additive and absolutely continuous on $G$ and hence the condition follows. 


\section{BIBLIOGRAPHY}

1. Casper Goffman, Real functions, Rinehart, New York, 1953. MR 14, 855.

2. W. E. Hartnett and A. H. Kruse, Differentiation of set functions using Vitali coverings, Trans. Amer. Math. Soc. 96 (1960), 185-209. MR 22 \#12195.

3. Henri Lebesgue, Sur l'intégration des fonctions discontinues, Ann. École Norm. (3) 27 (1910), 361-450.

4. Chr. Pauc, Review. MR 22 \#12195.

5. S. Saks, Théorie de l'intégrale, Monografie Mat., vol. 2, PWN, Warsaw, 1933; English transl., Monografie Mat., vol. 7, PWN, Warsaw, 1937.

6. Harvel Wright and W. S. Snyder, On the differentiability of arbitrary real-valued set functions. I, Trans. Amer. Math. Soc. 145 (1969), 439-454. MR 40 \#4416.

7. R. C. Young, Functions of $\Sigma$ defined by addition or functions of intervals in n-dimensional formulation, Math. Z. 29 (1928), 171-216.

Oak Ridge National laboratory, Oak Ridge, Tennessee 37830 